\title{
Search for plastic flow autowaves in ice by radio brightness temperature
}

\author{
G. S. Bordonskiy, A. A. Gurulev, A. O. Orlov, S. V. Tsyrenzhapov \\ Institute of Natural Resources, Ecology and Cryology SB RAS, Chita, 672090, Russia \\ E-mail: lgc255@mail.ru
}

\begin{abstract}
It has been found that, with plastic deformation in ice, anomalies of its microwave characteristics occur. It has been previously demonstrated that at plastic flow localized plastic flow autowaves are formed in them. Characteristic flow wavelengths were in the range of $0.5-2 \mathrm{~cm}$. In this work, to account for the anomaly of the microwave characteristics of ice, we searched for localized plastic flow autowaves in it. We measured radio brightness temperature of the ice cover at frequencies from 10 to $34 \mathrm{GHz}$ during significant daily fluctuations of the air temperature. As the direction of the temperature gradient in the ice cover depth changed fast, resulting in plastic deformation, we observed decrease of the radio brightness temperature by $10-15 \mathrm{~K}$. The data obtained confirm the existence of localized plastic flow autowaves in polycrystalline fresh-water ice.
\end{abstract}

\section{Introduction}

Normally, the characteristics of ice bodies are investigated in the static mode. Much less studied are the dynamic characteristics of ice, for example, in the process of its deformation. In [1, 2], anomalies of the microwave characteristics of ice formations were discovered, which were related to plastic deformation and emergence of plastic flow autowaves.

Plastic flow autowaves were previously discovered theoretically and experimentally for metals having crystalline structure [3, 4]. In accordance with the theory developed, in crystalline bodies plastic flow autowaves emerge at a macro level with characteristic wavelengths from 0.5 to $2 \mathrm{~cm}$. Their propagation rate is low: $\sim 10^{-5}-10^{-6} \mathrm{~m} / \mathrm{s}$. They represent alteration of regions with practically non-deformed crystalline structure and active regions in which plastic deformation develops. Flow autowaves were discovered on the surface of metal specimens using the speckle photography technique [5].

Novel representations of the ice structure open up possibilities of remote studies of internal structures changing in time in permafrost bodies exposed to external mechanical impacts. The objective of the present study was to obtain experimental evidence of the existence of plastic flow autowaves in a natural formation (ice cover) and to ascertain the possibility of their registration by the microwave radiometry method.

\section{Properties of ice crystals}

\subsection{Mechanical properties}

The unique quality of hexagonal ice Ih consists in the non-linear dependence between deformation and mechanical stress $(\sigma)$ for a monocrystal at relatively low stresses. This quality is especially noticeable at shifting of parts of the ice crystal along the crystal's basic planes. Shown in figure 1 are the results of the experiments described in a monograph [6]. It follows from figure 1 that, for example, in 
the region of stresses exceeding $0.45 \mathrm{MPa}$ for conditions (6), as deformation develops, it grows as the stress in the medium decreases. This means that negative differential viscosity emerges in the crystal.

In general terms, in the crystals of ice Ih, we may expect formation of dissipative structure at mechanical impacts. Relatively recently, wave structures were discovered, as noted above, in crystals of metals in certain phases of plastic deformation, which is confirmed by this assumption. In polycrystalline ice specimens, they may be expected to occur as fragments of a periodic structure, diffraction lattices due to the low rate movement. Such lattices effectively scatter radiation, if their period is close to the wavelength $(\lambda)$ of the electromagnetic radiation falling onto them. Respective wavelengths at normal fall of radiation onto the lattice plane should be in the range shorter than $2 \mathrm{~cm}$ (if the maximum wavelength of the flow waves is $2 \mathrm{~cm}$ ), in accordance with the formula of the diffraction lattice: $d \sin \varphi=k \lambda$, here $d$ - lattice parameter, $\varphi-$ the angle of the ray's deflection from the normal $(k=0,1,2, \ldots)$.



Figure 1. Dependence 'stress-deformation' for an ice monocrystal at a fixed deformation rate in the case of shifting along a basic plane. The crystal temperature is $-15^{\circ} \mathrm{C}$. The deformation rate: $1-1.3 \cdot 10^{-7} 1 / \mathrm{s}$; $2-3 \cdot 10^{-7} 1 / \mathrm{s} ; 3-7 \cdot 10^{-7} 1 / \mathrm{s} ; 4-13 \cdot 10^{-7} 1 / \mathrm{s} ; 5-$ $17 \cdot 10^{-7} 1 / \mathrm{s} ; 6-27 \cdot 10^{-7} 1 / \mathrm{s}[6]$.


Figure 2. Dependences of standard deviation values of phase $S \varphi$ (a) and intensity $S_{\mathrm{I}}$ (b) on frequency at different ice temperatures.
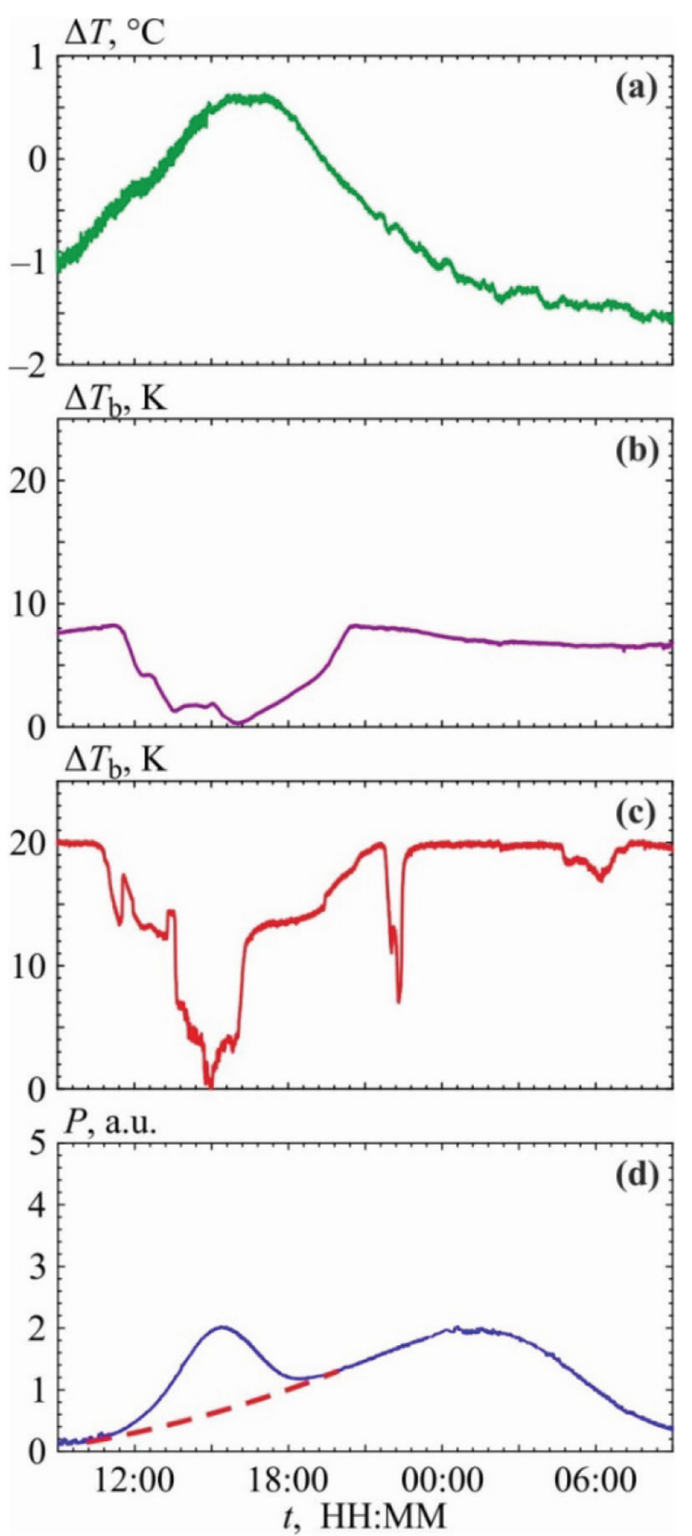

Figure 3. Measurement results for: (a) - vertical ice temperature gradient in the upper layer; (b), (c) - increment radio brightness temperatures and (d) - increment of power transmitted through the ice cover in parallel to the surfaces at the frequency of 13.4 GHz (at arbitrary units). 
Similar phenomena occur in other systems, for example, in electric circuits of the alternating current. If negative differential resistance emerges in them, this, under certain conditions, leads to generation of electromagnetic oscillations. Such systems are active: in them, supplied energy (for example, the direct current energy) is transformed into the energy of regular oscillations [7]. However, in glaciology this feature of ice has not been investigated, although structuring is well-known for dissipative systems in thermodynamics of irreversible processes [8].

\subsection{Electromagnetic characteristics at plastic flow}

Unlike metals, ice structures transmit electromagnetic radiation with wavelengths corresponding to the lattice period of plastic flow autowaves. This allows them to be detected by scattering of radiation on inhomogeneities in the volume of an ice specimen.

In [9], we conducted laboratory studies of the reflection coefficient from a block of ice cut from the ice cover of a fresh-water lake. Measurements were made at the frequencies of $12-13.5 \mathrm{GHz}$. Mechanic stresses in the block were generated by heating a cold specimen, in which due to the temperature difference, emergence of plastic flow autowaves was expected.

In this experiment, for the first time in a block of ice, inhomogeneities of a certain size and emergence of fluctuations of a reflected signal were discovered.

Shown in figure 2 are the results of measuring the fluctuation range of the phase and intensity of scattered microwave radiation. Out of these data, the mean value of the lattice parameter proved to be equal to $\sim 1.4 \mathrm{~cm}$, corresponding to the values predicted in $[3,4]$.

\section{Measuring radio brightness of the ice cover}

To view manifestations of flow waves in a natural environment, ice covers of fresh-water lakes were chosen. It is known that at the end of the freeze-up period cracks and ice break are observed in the ice cover due to emergence of intense mechanical stresses. They are caused by daily fluctuations in the ice temperature and reduction of its strength. These characteristics were used in searching for flow waves. In the experiment conducted, intensity of radiothermal radiation was measured at the frequencies from 10 to $34 \mathrm{GHz}$, depending on the electrophysical characteristics of the water-ice system and the presence of radiation dissipating inhomogeneities in the ice cover.

The measurement region was in the area with a sharply continental climate (Zabaikalsky krai (Transbaikalia), near the Yablonovy Range) at the altitude of $\sim 940 \mathrm{~m}$ asl. The region is characterized by sharp contrasts between the day and night air temperatures, which at the time of observations reached $20^{\circ} \mathrm{C}$. Measurements were made on the ice cover of Arakhley Lake with salt concentration of water $\sim 120 \mathrm{mg} / \mathrm{l}$. Thickness of the snow cover of the ice cover of the lake was $\sim 5 \mathrm{~cm}$. Those conditions generated noticeable variable mechanical stresses in the ice.

Microwave radiometers for the frequencies of 10, 21 and $34 \mathrm{GHz}$ with the bandwidth of $\sim 1-2 \mathrm{GHz}$ were installed onto the ice cover near the surface. The observation angle was selected to be $45^{\circ}$, polarization of the received radiation was horizontal. Simultaneously, microwave raying of the ice cover was conducted at the frequency of $13.4 \mathrm{GHz}$ in parallel to the ice surface as the equipment was being installed into the $0.5 \mathrm{~m}$ deepening in the ice cover. Ice thickness during the observations was $1.2 \mathrm{~m}$. The distance between the transmitter and the receiver was $40 \mathrm{~m}$. The experiments lasted several days to achieve favorable conditions for emergence of plastic deformation. On the days when anomalies of microwave characteristics were observed (28.02-01.03.2019), the air temperature varied during a day in the range of $-20 \ldots 0^{\circ} \mathrm{C}$.

The results of measuring the vertical temperature gradient $(\Delta T)$ in ice by measurements at two depth values from the ice surface $4 \mathrm{~cm}$ and $10 \mathrm{~cm}(\Delta T=T(4 \mathrm{~cm})-T(10 \mathrm{~cm}))$ are shown in figure $3 \mathrm{a}$. In figure $3 \mathrm{~b}$, the results of measuring radio brightness temperature $\left(T_{\mathrm{b}}\right)$ at the frequencies of 21 and $34 \mathrm{GHz}$ are demonstrated.

The extremum of the transmitted radiation power $(P)$ against the background of slow daily variations was observed at the frequency of $13.4 \mathrm{GHz}$ simultaneously with the minimum of the radio brightness temperature - figure $3 \mathrm{~d}$. The dashed line shows the change in signal by analogy with other days. At the frequency of $10 \mathrm{GHz}$, no essential changes in the radio brightness temperature were recorded. 


\section{Discussion of results}

The discovered features of the microwave signals may be explained by emergence of plastic flow autowaves in polycrystalline ice. Anomalies were registered at the wavelengths of electromagnetic radiation in ice $\left(\lambda_{\text {ice }}\right)$. constituting $0.5-1.4 \mathrm{~cm}: \lambda_{\text {ice }}=\lambda_{0} /\left(\varepsilon^{\prime}\right)^{1 / 2}$, where $\lambda_{0}-$ the wavelength in free space, $\varepsilon^{\prime}=3,15$ is the real part of relative dielectric permittivity of ice at microwaves. The obtained values of wavelengths of electromagnetic radiation correspond to the characteristics of plastic autowaves which formed diffraction lattices [3, 4].

In radiometric measurements, emergence of plastic flow waves led to radiation scattering of the ice cover and decrease of the radio brightness temperature. At the frequency of $34 \mathrm{GHz}\left(\lambda_{\text {ice }} \sim 0.5 \mathrm{~cm}\right)$, we observed complex behavior of the microwave signal in time (figure 3c). At certain moments of time, abrupt change of radio brightness temperature were recorded. At radio raying of the ice cover, at the distance of $40 \mathrm{~m}$, microwave signal anomalies were discovered simultaneously with radiometric measurements, indicating emergence of the effect on a large-size ice field. This feature reaffirms the impact of thermal stress which caused plastic deformation on the microwave characteristics of ice, in a floating ice cover.

Saline ice of a soda lake was also investigated, with salt concentration in water being $35 \mathrm{~g} / 1$ and in ice about $1 \mathrm{~g} / \mathrm{kg}$. For such an ice body, flow autowaves were not discovered at microwave measurements. It is likely that plastic deformation of such ice occurred along liquid interlayers of non-freezing solutions, not along its crystals.

\section{Conclusions}

1. Microwave measurements revealed occurrence of plastic flow autowaves in fresh-water ice with characteristic values of alternating plastically deformed and resiliently deformed areas $\sim 0.5-1.4 \mathrm{~cm}$. Decrease of the radio brightness temperature of freshwater ice cover, caused by daily variation of the air temperature (from 0 to $-20^{\circ} \mathrm{C}$ ), was $10-15 \mathrm{~K}$ for the ice cover with little snow.

2. Appearance of plastic flow autowaves allows observation of the ice behavior at the early stage of its movement, when plastic deformation emerges. Measurements of the characteristics of this process are possible using the microwave radar method (by the increment of the reflection coefficient) and the radiometric method (by decrease of the radio brightness temperature).

\section{References}

[1] Bordonskiy G. S., Krylov S. D., Amorphization of ice under mechanical stresses, Technical Physics Letters, 2017, Vol. 43, pp. 983-986.

[2] Bordonskiy G. S., Reasons for the formation of incoherent additional microwaves in fresh ice under plastic deformation, Technical Physics, 2016, Vol. 61(8), pp. 1250-1255.

[3] Zuev L. B., Barannikova S. A., Evidence for the existence of localized plastic flow autowaves generated in deforming metals, Natural Sciences, 2010, Vol. 02(05), pp. 476-483.

[4] Zuev L. B., Autowave processes of the localization of plastic flow in active media subjected to deformation, Physics of Metals and Metallography, 2017, Vol. 118(8), pp. 810-819.

[5] Zuev L. B., Gorbatenko V.V., Pavlichev K.V., Elaboration of speckle photography techniques for plastic flow analyses, Measurement Science Technology, 2010, Vol. 21(5), 054014, 5 p.

[6] Bogorodskiy V.V., Gavrilo V.P., Ice. Physical Properties. Modern Glaciology Techniques, Leningrad: Hydrometeoizdat, 1980, $384 \mathrm{p}$.

[7] Khalil H. K., Nonlinear Systems, New York: Prentice Hall, 2002.

[8] Kondepudi D., Prigogine I., Modern Thermodynamics: From Heat Engines to Dissipative Structures, 2nd Edition, New York: John Wiley and Sons, 2014.

[9] Bordonskii G,S., Gurulev A. A., Plastic Flow Autowaves in Freshwater Ice As Manifested by Microwave Reflection Measurements, Technical Physics Letters, 2019, Vol. 45(3), 285-287. 